\title{
Aplicación de la Tecnología de la Información y Comunicación en la enseñanza de anatomía para estudiantes de enfermería ${ }^{1}$
}

\author{
Viriam Leiva Díaz ${ }^{2}$ \\ Elena Mora Escalante ${ }^{3}$
}

Institución: Universidad de Costa Rica

\section{CÓMO CITAR}

Leiva, V. y Mora, E. (2014). Aplicación de la Tecnología de la Información y Comunicación en la enseñanza de anatomía para estudiantes de enfermería. Rev. Enfermería Actual de Costa Rica, 26, 1-13. Recuperado de: 〈http://www.revenf.ucr.ac.cr/tic.pdf> ISSN 1409-4568

\section{RESUMEN}

Se presenta los resultados de la primera etapa de un estudio que es parte del proyecto de investigación $\mathrm{n}^{\circ}$. 087 titulado "Aplicación de tecnologías de información y comunicación en la gestión docente de la enseñanza de anatomía para estudiantes de enfermería", el cual pertenece a la Escuela de Enfermería de la Universidad de Costa Rica. El estudio se basa en los fundamentos de la Investigación cuantitativa descriptiva. La población participante estuvo constituida por el alumnado inscrito en el curso de Anatomía para enfermería (67 estudiantes del recinto de Golfito), de los cuales participaron 22 de ellos y ellas en el estudio. Este curso se imparte durante el primer ciclo del plan de estudios de la carrera de Licenciatura en Enfermería. Para recolectar información, se aplicó un cuestionario. El estudio evidenció que el uso de la Tecnología de la Información y Comunicación (TIC) en la enseñanza de la anatomía para enfermería contribuye al proceso del aprendizaje significativo; además, la relación que se estableció en el desarrollo de los conceptos teóricos de anatomía en situaciones concretas de la práctica de la enfermería fungió como motivador extrínseco fundamental para concretar los objetivos del curso, proceso del que surgió la segunda parte de la investigación planteada: el análisis de la efectividad de este curso en la formación del alumnado que cursa el último año de la carrera. Se concluye que el uso de las TIC en los diversos cursos de la carrera de Licenciatura en Enfermería representa una estrategia novedosa que beneficia el proceso de enseñanza- aprendizaje; sin embargo, es claro que no sustituye al docente, es más, exige no solo mayor dominio de parte de este en cuanto a las nuevas tecnologías de información, sino manejo de la cantidad de datos, capacidad en la selección y calidad de la información de la que dispone el estudiantado para facilitar el aprendizaje significativo.

Palabras clave: anatomía, aprendizaje-significativo, enfermería, tecnología-de-información

\footnotetext{
${ }^{1}$ Fecha de recepción: 22 de octubre del 2013

Fecha de aceptación: 10 de enero del 2014

${ }^{2}$ Enfermera. Licenciada en Psicología. Magister en Psicopedagogía. Profesora Catedrática de la Universidad de Costa Rica. Escuela de Enfermería. Correo electrónico: viriaml@gmail.com

${ }^{3}$ Enfermera en Salud Mental y Psiquiatría. Magister en Terapia Familiar Sistémica. Profesora Adjunta de la Universidad de Costa Rica.

Escuela de Enfermería. Correo electrónico: moresca21@hotmail.com
} 


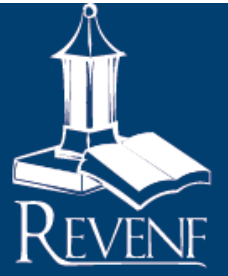

\section{Application of Information Technology and Communication in the teaching of anatomy for nursing students ${ }^{1}$}

Viriam Leiva Díaz ${ }^{2}$

Institution: University of Costa Rica

Elena Mora Escalante ${ }^{3}$

\section{Cited}

Leiva, V. y Mora, E . (2014). Application of Information Technology and Communication in the teaching of anatomy for nursing students. Rev. Enfermería Actual en Costa Rica, 26, 1-13. Available: <http://www.revenf.ucr.ac.cr/tic.pdf> ISSN 1409-4568

\section{ABSTRACT}

The results of the first stage of a study that is part of the research project $n^{\circ} 187$ is presented, it belongs to the School of Nursing at the University of Costa Rica. The study was based on the fundamentals of descriptive and quantitative research. The participant population consisted of students enrolled in the Anatomy course for nursing (67 students Enclosure Golfito), of which 22 of them participated in the study. The course is given during the first cycle of the curriculum of Nursing. To collect data, a questionnaire was applied. The study showed that the use of Information Technology and Communication (TIC) in the teaching of anatomy to nursing contributes to meaningful learning process, in addition, the relationship established in the development of theoretical concepts of anatomy in specific situations of nursing practice served of extrinsic motivator and essential to achieve the objectives of the course, in the second phase of the proposed investigation arose: analysis of the effectiveness of this course in the formation of the pupils enrolled in the final year of the race. We conclude that the use of TIC in the various courses of the curriculum of nursing represents a novel strategy that benefits the teaching-learning process, however, clearly does not replace the teacher, demand great domain as part of the new technology information, handling of amount data, selection capacity and quality of the information available to the students.

Keywords: anatomy, information-and-communication-technology, meaningful-learning, nursing

\footnotetext{
${ }^{1}$ Date of receipt: October 22, 2013

Date of acceptance: January 10, 2014

${ }^{2}$ Nurse. Degree in Psychology. Master in Psychopedagogics. Professor at the University of Costa Rica. School of Nursing. E mail: viriaml@gmail.com

${ }^{3}$ Nurse in Mental Health and Psychiatry. Master in Systemic Family Therapy. Professor at the University of Costa Rica. School of Nursing. E mail: moresca21@hotmail.com
} 
.

\section{INTRODUCCIÓN}

El estudio de la anatomía es indispensable para comprender los fenómenos y procesos fisiológicos, bioquímicos y clínicos, relacionados con la salud del ser humano como un todo (Falcó, 2005); por lo tanto, es imprescindible que el estudiantado de enfermería adquiera conocimientos profundos acerca de las estructuras, órganos y sistemas del organismo normal, con el fin de que identifiquen alteraciones y o problemas en las estructuras, los cuales deben ser solucionados por el profesional desde un abordaje holístico de calidad.

A pesar de su relevancia, hoy a dichos conocimientos se les asigna tiempos cortos para su aprendizaje, de modo que urge implementar nuevas estrategias para presentar y explicar los contenidos de forma organizada, concisa y atrayente (usando imágenes y ejemplos), tarea que es posible si se muestra claramente la relevancia clínica y la utilidad práctica de la materia, cuyo objetivo sería facilitar la comprensión teórica de los temas anatómicos. El uso de elementos tecnológicos como el entorno virtual y otros medios computacionales, junto con elementos pedagógicos como el principio del aprendizaje significativo, solventan los problemas descritos en el párrafo anterior, tal como lo afirma Quispe (2005): "la voz del profesor y los textos ya no son los únicos medios por los que los educandos se aproximan al conocimiento, estos lo hacen desde y con la tecnología” (p. 1).

Un ejemplo de lo mencionado, lo refieren Inzunza y Bravo (2005), en una investigación acerca del uso de programas computacionales para la docencia en anatomía humana en la cual destacan que las tecnologías computacionales sirvieron como medios autoinstructivos modernos, adecuados para responder al aumento del número en el alumnado y para mejorar el conocimiento teórico de la materia de anatomía. En relación con el tema, Bouza y Guardado (1999) afirman que el manejo estratégico de los recursos informáticos mejora considerablemente la misión de la institución y fomenta la disponibilidad y acceso al conocimiento; en sus aportes destacan la visión actual del apoyo que la tecnología de la información y comunicación (TIC) brinda a la enseñanza universitaria de diversas materias, cuyo reto es desarrollar investigación y experiencias docentes, relacionadas con la maximización y potenciación de los recursos que brindan para el aprendizaje significativo del estudiantado (Salinas, 2004; Cardozo, 2008).

Otro aporte que refuerza lo anterior es el que brinda Azpilcueta (2002) en un estudio acerca del uso de herramientas computacionales, multimedia e internet como una alternativa educativa en las universidades; el investigador confirmó que este tipo de herramientas influyen de manera positiva tanto en la motivación del alumnado, como en su retención; resultado que aumenta la calificación, reduce la diferencia entre el promedio más alto y el más bajo y disminuyó el tiempo empleado para cubrir la parte teórica de un curso en casi un $50 \%$ en uno de los casos observados.

El aporte práctico de las TIC beneficia en dos áreas: por un lado, en la docencia, dado que se trata de adaptar la escuela a la realidad y facilitar al profesorado su tarea de orientador, diseñador, facilitador, comunicador, coordinador, asesor y evaluador del aprendizaje; por el otro, se ayuda a las y los estudiantes, puesto que incentiva un aprendizaje más activo y participativo, durante las clases virtuales a través de toda la etapa de ejecución y comunicación del trabajo (Cardozo, 2008). Dado lo anterior, las TIC brindan un enorme potencial en cuanto a posibilidades en la esfera educativa; no obstante, es necesario recalcar que la implementación de las TIC no significa reemplazar al profesorado, por el contrario facilita el proceso de enseñanza-aprendizaje, aparte de que por su flexibilidad promueven una comunicación bilateral más intensa entre el docente y dicente, de modo que se convierte en una herramienta motivadora con la que las y los estudiantes adquieren datos provenientes de 
ISSN $1409-4568$

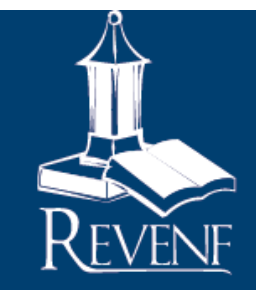

Revista Electrónica Eneremeria Actualen costa Rica

www.revenf.ucr.ac.cr

diversas fuentes en tiempo real, mientras que explora el mundo virtual, a través de la escucha, la vista, sentidos que potencian el aprendizaje y que garantizan una mayor difusión en diversos cursos respecto de la enseñanza tradicional (Gurdián, 2001; Dussel y Quevedo, 2010).

En relación con lo anterior, Meléndez (2009) refiere que la mediación virtual es una herramienta complementaria de la enseñanza, una forma de docencia universitaria alternativa, más flexible e interactiva por la cantidad de recursos con que cuenta el profesorado universitario (imágenes, audio, videos, música, foros y de chats), respecto de lo cual destaca que,

la tecnología no sustituye la docencia, sino que moldea nuevos modos....tiene como objetivo prioritario promover una docencia universitaria alternativa, más flexible e interactiva (p.1); finalmente, afirma que en su práctica cotidiana como docente, el uso de las TIC le ha permitido un aumento en el rendimiento de los estudiantes, debido a su flexibilidad que intrínseca (p1).

En el caso de la Universidad de Costa Rica, la institución cuenta con una plataforma educativa MOODLE (LMS, Learning Management Systems), la cual se sustenta en los enfoques del constructivismo en pedagogía y en el aprendizaje colaborativo (Baños, 2007) en el que, como se ha mencionado, se puede emplear tecnologías: transmisivas, interactivas, colaborativas (Galbis, 2002), aparte de que se logra la transferencia de la información, la adquisición de habilidades y un cambio del modelo mental del estudiante, tal como lo manifiesta Marquéz (2004),

el uso innovador de todas las prestaciones de las TIC en el marco de una cultura colaborativa e integradora son tan importantes que nos permiten dibujar un nuevo paradigma de enseñanza basado en unos planteamientos cognitivistas y socioconstructivistas del aprendizaje, que promueva un aprendizaje a partir de la búsqueda, la experimentación, la interacción (con recursos y personas), la asimilación y aplicación de los conocimientos (y no su mera memorización). En definitiva, se trata de pasar de una enseñanza dividida en asignaturas centrada en el profesor ("teaching by telling") a una enseñanza más interdisciplinar y centrada en el alumno ("learning by doing"), en la que lo más destacable es el aprendizaje de las y los alumnos (lo que descubren, lo que piensan, lo que dicen, lo que hacen, lo que organizan con autonomía...) con la ayuda, orientación y mediación del profesor, que actúa también como organizador de los aprendizajes, atendiendo a sus estilos de aprendizaje (p.1).

De este marco se desprende el objetivo que fue evaluar el uso de las TIC en la enseñanza de la anatomía para enfermería en el estudiantado del recinto de Golfito de la Universidad de Costa Rica.

\section{MATERIALES Y MÉTODOS}

La población comprendida en esta investigación correspondió a los estudiantes matriculados en el curso Anatomía humana para enfermería, impartida en el Ier ciclo del plan de estudios de la carrera de Licenciatura en Enfermería, integrado por un total de 67 estudiantes de enfermería del recinto de Golfito.

Se desarrolló una investigación de aula (Martínez, 2000; Hernández, Fernández y Baptista 2007), basada en los fundamentos de enfoque cuantitativo de tipo descriptivo, la cual se inicia con la fase indagatoria de la 
temática para la consecución del primer objetivo; continúa con la fase de implementación para la consecución del segundo objetivo y finaliza con la fase de evaluación, que corresponde al tercer objetivo específico.

Para evaluar la experiencia obtenida, se utilizó la técnica de cuestionario autoadministrado que comprende los siguientes apartados: expectativas del curso, temas desarrollados, metodología, métodos y técnicas utilizadas, mediación pedagógica de los contenidos y materiales, bibliografía y recursos utilizados para facilitar el aprendizaje, condiciones del ambiente de aprendizaje y opinión del alumnado acerca de las fortalezas y debilidades.

Los datos fueron procesados utilizando el paquete estadístico excel y posteriormente se analizaron las estadísticas descriptivas.

\section{Consideraciones éticas}

Coincide con los principios que establece la bioética entre ellos la confidencialidad y el consentimiento informado; los datos recopilados se usarían únicamente para el diseño e implementación de un plan de acción para el mejoramiento del curso.

\section{RESULTADOS}

De los 22 estudiantes, la edad de 19 de ellos oscilan entre los 19 y 22 años: la moda correspondiente a la edad es de 21 años (11). En cuanto al género, 14 son mujeres y 8 son varones.

La gran mayoría (19) de las y los estudiantes participantes son solteros. En cuanto al año de ingreso a la carrera, 15 lo hicieron en el año 2009 y 7, en el 2010.

En lo que respecta a la beca que disfruta el alumnado, se destaca que todos (22) disfrutan becas que van desde la beca 5 hasta la beca 11 (en esta última se agrupa la gran mayoría (16)).

En cuanto al curso de Anatomía para enfermería, 14 estudiantes lo matricularon en el año 2009 y 8 en el 2010: todos ellos y ellas matricularon el curso una sola vez.

En cuanto a la claridad de los objetivos de aprendizaje planteados en el curso de Anatomía para enfermería, 19 de los estudiantes refirieron que fueron claros (14) y muy claros (5), mientras que el resto de las y los participantes contestaron que fueron más o menos claros (2) y un estudiante refirió no conocer los objetivos.

En cuanto a los recursos empleados para el desarrollo de los contenidos del curso de Anatomía para enfermería, los estudiantes afirman que las herramientas más actualizadas fueron el examen y las tareas con igual cantidad de opiniones (19), seguida por el uso del foro (17) y el correo del aula virtual (14), mientras que en el último lugar se encuentran el chat y el wiki.

Las y los estudiantes están muy de acuerdo (8) con que el ambiente virtual del curso facilitó una apropiación más fácil de los contenidos del curso; por ejemplo, el participante 11 agregó: "El curso fue realmente útil y la metodología utilizada fue excelente", mientras que otro expresó "Es uno de los cursos más importantes de 
la carrera. Los contenidos y los métodos en que se desarrollan fueron muy buenos" (sujeto 8). Cabe destacar que otro grupo (16) estuvo de acuerdo y medianamente de acuerdo, posición que se resume en la siguiente opinión "Es muy buena la forma de educación a distancia, pues el tiempo alcanza un poco más; sin embargo, no es completamente satisfactoria" (sujeto 21).

En cuanto a la opinión de las y los estudiantes acerca de si las actividades virtuales propuestas en el curso de Anatomía para enfermería facilitaron su aprendizaje, 9 participantes se mostraron muy de acuerdo; 5, medianamente de acuerdo y 5 , de acuerdo con esta aseveración.

Respecto del ambiente de aula virtual interactivo, 17 de las y los estudiantes se ubicaron en los criterios de muy de acuerdo y de acuerdo, dado que facilitó la comunicación e interacción constante entre el grupo de iguales y la docente, así lo demuestra el siguiente comentario: "La comunicación estudiante-profesora fue excelente" (sujeto 5). También, 21 estudiantes consideraron que las clases virtuales facilitaron el rol del docente como comunicador, coordinador y orientador del proceso de aprendizaje; esa misma cantidad opinó que el uso del aula virtual incentivó un contacto más frecuente y expedito con la docente, en comparación con otros docentes de cursos regulares; sin embargo, esa información es contradictoria, debido a que 17 estudiantes consideraron que el número de encuentros presenciales con la docente no fue suficiente, lo cual se constata en la siguiente opinión: "Muy buena profesora, pero no me gustan las aulas virtuales y sería bueno más contacto con la profesora y mayor tiempo en los laboratorios" (sujeto 10).

En cuanto al manejo de las temáticas, el total de estudiantes destacó el dominio temático de la docente y la participación de los asistentes del curso, quienes facilitaron el proceso de aprendizaje, lo cual se desprende de las opiniones a continuación: "Una profesora que conoce bien los temas tratados" (sujeto 1), "El dominio de temas por parte de la docente" (sujeto 16), "Hace énfasis en puntos importantes de la enfermería" (sujeto 11).

La mayoría del estudiantado participante (21) manifestó que esta estrategia de aprendizaje en que media el uso de tecnología de información mantuvo su interés y motivación, en comparación de los medios convencionales de enseñanza, algunos comentarios así lo manifiestan : "Aprendí a interactuar con otro ambiente" (sujeto 3), "en contacto constante con la profesora" (sujeto 5), "muy interactivo"( sujeto 22). Esos 21 estudiantes agregaron que la posibilidad de introducir y subir al aula virtual material compartido por sus compañeros (as) fue relevante para el aprendizaje de los contenidos del curso, información que colige de las actividades "fortalezas", dentro de las que se encuentran el "aula virtual", la "interacción virtual" y, finalmente, los "estudiaderos".

En relación con el enfoque del curso centrado en la importancia de los contenidos abordados hacia enfermería, 21 participantes manifestaron estar muy de acuerdo y de acuerdo con este, debido a que fueron útiles en sus prácticas clínicas y módulos de enfermería; inclusive más de la mitad de ellos (15) manifiestan que aumentó su capacidad de relacionar los temas de los módulos, tales como patologías o procesos mórbidos; luego, toda la población afirmó la utilidad de los contenidos para comprender los contenidos desarrollados en otros cursos de enfermería, lo cual se manifiesta en los siguientes comentarios: "Una excelente base para los demás cursos y enfermería en sí" (sujeto 4); "En síntesis, el curso es bueno: en él se adquieren conocimientos para nuestra profesión"(sujeto 2). 
Por otra parte, respecto de si el aporte práctico del curso de anatomía fue suficiente, los participantes se mostraron medianamente de acuerdo y muy de acuerdo, incluso, 17 de ellos expresaron que la cantidad de horas de práctica no fueron suficientes en todas las unidades desarrolladas en el curso, así lo manifiesta una de las estudiantes: "en general el curso me pareció excelente; sin embargo, tuvimos poco tiempo en los laboratorios" (sujeto 7); otra alumna expresó un comentario en la misma dirección: "Pocas horas de práctica"(sujeto 19). Se puede resumir la posición de las y los estudiantes con la siguiente opinión: "El tiempo de laboratorio fue reducido: si hubiésemos tenido el laboratorio, hubiera sido más provechoso; sin embargo, me siento satisfecho con el curso" (sujeto 13).

El trasladarse a la sede Rodrigo Facio para el uso de los laboratorios de Anatomía fue un aspecto positivo para la mitad de los estudiantes (12); ya fuera a favor o en contra, es innegable el impacto que produjo en ellos viajar desde el recinto de Golfito hasta la sede Rodrigo Facio, debido a que "Viajar tantas horas para poder realizar los laboratorio es cansado"(sujeto 2); "En caso contrario, habría menos interacción con los cuerpos anatómicos" (sujeto 15); "El viaje tan lejos realizado afecta un poco la concentración del estudiante por el cansancio" (sujeto 20). De las opiniones anteriores, se colige que las y los estudiantes consideran necesario contar con un laboratorio de Anatomía en el recinto: "Se necesita un laboratorio de anatomía en el recinto" (sujeto 4); otra de las personas participantes afirma que "sería de gran utilidad que en el recinto se pudiera contar con un pequeño laboratorio"(Sujeto 20), dado que de la totalidad del estudiantado considera que, comparado este curso con otros cursos de servicio, este es uno de los más relevantes; por tanto, debido a las dificultades, todos opinan que lo mejor sería el curso presencial.

Finalmente, en cuanto a la evaluación de los aprendizajes, la opinión de las y los estudiantes estuvo dividida: más de la mitad estuvo de acuerdo (17) y destacó aspectos como el aula virtual, la interacción virtual, la mediación virtual, los videos de las partes anatómicas, tal como lo comenta una persona participante: "El curso fue realmente útil y la metodología utilizada fue excelente"(sujeto 1); no obstante un pequeño grupo se manifestó en desacuerdo, opinión que se ejemplifica con la siguiente expresión: "Muy buena profesora, pero no me gustan las aulas virtuales y sería bueno más contacto con la profesora y mayor tiempo en los laboratorios" (sujeto 10). En cuanto al material bibliográfico, todos los estudiantes lo evaluaron como adecuado y actualizado del que destacaron "el material del contenido" (sujeto 16) "libro de texto" (sujeto 5).

Aparte de todo el insumo teórico-práctico recibido, los participantes rescatan la interiorización de valores necesarios para su quehacer profesional, tales como liderazgo, respeto y pasión por la enfermería.

\section{DISCUSIÓN}

Para iniciar este apartado, es necesario comenzar con el referente que enmarca esta investigación de aula, el aprendizaje significativo, el cual se comprende como aquel que requiere motivación por parte del estudiante y que se manifiesta en ese deseo de aprender significativamente, como lo plantea Del Prado (2011): "Para que se produzca el aprendizaje significativo es necesario que el alumno sea capaz de dar sentido a lo que aprende" (p.1).

Además para que se generen nuevas ideas, estas deben basarse en conocimientos previos, los cuales tienen que ser claros y específicos, tal como lo menciona Bolívar (2009): 
El aprendizaje del alumno depende de la estructura cognitiva previa que se relaciona con la nueva información, entendiendo por "estructura cognitiva", al conjunto de conceptos, ideas que un individuo posee en un determinado campo del conocimiento, así como su organización (p. 1).

De lo anterior se colige que la forma como se enseña una determinada materia es fundamental para la construcción de ese saber y base para la preparación de nuevas estructuras cognitivas que constituyen una profesión como la enfermería.

El docente debe contribuir con la construcción del verdadero saber en el estudiantado, entendiendo este como aquel que se mantiene en el tiempo, el que puede establecer enlaces con otro saber; como lo manifiesta Ballestero (2000), para que se produzca un verdadero saber el docente debe aportar estrategias didácticas que permitan ese proceso:

Auténtico aprendizaje, es decir; un aprendizaje a largo plazo y que no sea fácilmente sometido al olvido, es necesario conectar la estrategia didáctica del profesorado con las ideas previas del alumnado y presentar la información de manera coherente y no arbitraria, "construyendo", de manera sólida, los conceptos, interconectando los unos con los otros en forma de red del conocimiento.

En resumen, Ballestero (2000) se refiere a que como docentes es necesario facilitar un aprendizaje cognitivo y metacognitivo a la vez, considerando el conocimiento previo, entendido, como las ideas previas que pueden o no distorsionar el conocimiento que se integra al nuevo saber y comprendiendo que las ideas previas funcionan como marcos conceptuales que dirigen y orientan el procesamiento de la información que se estudia en las diversas fuentes, ya sea en libros de texto o las mismas explicaciones del docente (Campanario y Otero, 2000).

Lo anterior es fundamental no solo en el proceso de asimilación y acomodo de nuevos datos, sino en el proceso de interrelación que se da dentro del entorno de aula, dado que esas ideas previas inciden en las observaciones y las interpretaciones de lo observado; tales procesos son fundamentales para la presente investigación en cuanto a la resistencia mostrada por el estudiantado acerca de la necesidad de estar presente en el aula. El estudiantado que cursó la asignatura está acostumbrado a una relación de presencialidad frente al docente (física) y no es extraño entender cómo, a pesar de que hay satisfacción del curso, y de que se tuvo una presencia virtual en la red en términos de tiempo mayor que en los cursos corrientes, existe resistencia al cambio. Campanario y Otero (2000) resumen que,

parece claro, pues, que las ideas previas son resistentes al cambio. El resultado es que los alumnos mantienen dos esquemas de conocimientos. Por una parte, estarían sus conocimientos académicos sobre fenómenos, teorías, leyes, fórmulas y métodos para resolver problemas. Estos conocimientos académicos son útiles en el medio escolar dado que sirven para resolver ejercicios y para aprobar los exámenes tradicionales. Por otra parte, los alumnos mantienen muchas veces su arsenal de ideas previas, que son útiles para entender la realidad y para interaccionar con el medio que les rodea (p. 157). 
Tal como se infiere, al incluir estrategias novedosas, el docente debe de tomar en cuenta que la visión docente puede contribuir a mejorar el proceso de apropiación de un saber, aunque no debe olvidar el riesgo de resistencia que dichos esquemas de conocimiento pueden originar en el estudiantado respecto de la forma en que debe establecerse la relación docente - dicente. La anterior consideración es muy relevante para este trabajo, dado que al implementar las TIC, las y los estudiantes manifestaron (como se detalla en el apartado anterior) su relevancia en cuanto a facilitar su proceso de aprendizaje; aunque se esperaba una mayor presencia docente, la situación mejoró con el uso de la plataforma de MOODLE (Cole y Foster, 2008), dado que el tiempo horas clase no excede las cuatro horas semanales.

Otro aspecto destacable es que el aprendizaje obtenido no solo fue teórico; por ende, ante un cambio de esquema se detectó cierta resistencia qué generó desequilibrio y conflicto; no obstante se restableció el equilibrio y se produjo mayor apertura en las y los estudiantes en cuanto al uso de estrategias metodológicas innovadoras.

Otro elemento que debe mencionarse es la motivación mostrada por parte del estudiantado hacia el curso que se constituyó no solo en la causa del aprendizaje sino también en el aprendizaje que se gestó también en el curso. La disposición de los contenidos en el programa de curso, así como las distintas estrategias metodológicas aplicadas en el curso favorecieron el aprendizaje por parte del estudiantado y a la vez generaron una actitud favorable y una disposición subjetiva respecto de los contenidos del curso; es decir, total motivación, uno de los aspectos fundamentales en el aprendizaje, tal como lo resume Bolívar (2009):

El ser humano tiene la disposición de aprender, de verdad, solo aquello a lo que le encuentra sentido o lógica. El ser humano tiende a rechazar aquello a lo que no le encuentra sentido. El único auténtico aprendizaje es el aprendizaje significativo, el aprendizaje con sentido. Cualquier otro aprendizaje será puramente mecánico, memorístico, coyuntural: aprendizaje para aprobar un examen, para ganar la materia, etc. (p. 3).

Una de las estrategias para generar este aprendizaje significativo, más allá de completar un examen o ganar un curso, se deseaba relacionar los contenidos directamente con la profesión de enfermería; es decir, no desvincular dicho curso teórico con otros posteriores, sino que se interrelacione los contenidos con situaciones propias de la profesión, máxime si se considera que el contenido de este curso es un común denominador de casi todas las disciplinas del área de la salud y que las competencias de cada profesión deben definir las necesidades del saber según su área; lo anterior se puede ejemplificar con lo manifestado por Falcó (2005): "Se debe reconocer que no son iguales las competencias de las diversas profesiones en ciencias de la salud y que las necesidades de formación general entre titulaciones pueden ser diferentes" (p. 2); por ende, una de las fortalezas de la investigación fue el replanteamiento del curso, cambio que se evidencia en la descripción que desde enfermería se le dio, explícito en el syllabus del curso en el que se manifiesta que,

tiene como objetivo construir conocimientos de las estructuras, órganos y sistemas del organismo normal, que permitan integrar la función de enfermería en la identificación de las necesidades de las personas y poder brindar una atención holística y de calidad. Busca la aplicación de los conceptos teóricos a situaciones prácticas concretas, como en el examen físico de la persona realizado por el profesional de enfermería (p.p.1-2) 


\section{Revista Electrónica Enfermeria Actual en costa Rica}

La idea anterior plantea como objetivo generar un aprendizaje significativo, el cual comprende primero cómo aprende el alumnado, identificando antes, cómo construye el conocimiento, de lo cual se concluye que para aprender, el alumnado debe comprender y entender lo que se le enseña, lo que no significa no esforzarse o no estudiar. Dado que el clima en el aula es trascendental; hay que promover la motivación a través del requisito básico del aprendizaje significativo, considerado un aprendizaje a largo plazo, resultado que espera el docente y el estudiantado de los materiales aprendidos que se interioricen durante un lapso prolongado (meses o, incluso años), dado que serán indispensables en futuros cursos, prácticas clínicas y, por qué no, en la futura vida profesional del alumnado (Ausubel, Novak, Hanesian, 1978). Como lo resume Falcó (2005):

La asignatura de Estructura y función del cuerpo humano, troncal de primer curso, tiene por objetivos básicos introducir al alumno en el conocimiento de la anatomía y la físiología del cuerpo humano y asegurar el aprendizaje de contenidos previos necesarios para estudiar patología y cuidados de enfermería, en segundo y tercer curso (p. 211).

Durante el proceso, la participación de la docente fue esencial por su formación como enfermera y su capacitación en el proceso del aprendizaje significativo, así por como modificó la estructura tradicional en la que se brindaba la materia, en el cual introdujo no solo la comprensión de los contenidos teóricos de las partes anatómicas y planteó el establecimiento de conexiones entre los conceptos, estructuras y su interrelación con el campo de conocimiento, procedimiento con el que se extrapola la información aprendida a otra situación o contexto diferente, de modo que el aprendizaje es un aprendizaje real y a largo plazo, tal como se describe en el syllabus del curso:

Aprender la estructura y función normal de los órganos y sistemas del cuerpo humano, para transferir luego el conocimiento anatómico obtenido al ser humano vivo y operante. Aplicar los conceptos teóricos a situaciones prácticas concretas, como por ejemplo en el examen físico de la persona realizado por el profesional de enfermería. Investigar la aplicación de los conceptos anatómicos en los diferentes procedimientos que realiza el/la profesional de enfermería (p. 3).

Con este trabajo, quedó claro que para que se dé un aprendizaje significativo el proceso principal debe ser facilitar la integración de los conocimientos, razón por lo que se crearon situaciones de aprendizaje con las que el alumnado aplicó lo que sabía y construyó nuevos saberes a partir de ejemplos claros, transparentes, ilusionantes, estimulantes y positivos para el aprendizaje. La teoría que aporta el curso fue fundamental para fortalecer el conocimiento del curso y emplearlo en sus prácticas clínicas y módulos de enfermería, así como para la comprensión de otros cursos de la carrera de Licenciatura en Enfermería. El concretar este logro se debe al uso de las TIC en el planteamiento y desarrollo de este curso dado que, como lo menciona Bolívar (2009), el uso del ordenador trabaja como un elemento motivacional, brinda la posibilidad de avanzar al ritmo de cada estudiante, fomenta el trabajo en equipo e introduce un cambio en la visión tradicional de la relación docente - alumno, máxime que, como lo menciona González (2011),

lo más importante en este proceso de transformación de la docencia es la superación de una interacción lineal profesor - estudiante o una bidireccional entre estudiantes, por una relación múltiple en la que el uso de herramientas TIC constituye la fuente principal del conocimiento (p. 9). 
-

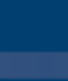

\section{Revista Electrónica Enfermería Actual en costa Rica}

www.revenf.ucr.ac.cr

Tal como se lee en la cita, la opinión de las y los estudiantes respecto del uso de las tecnologías de información fue positiva, puesto que mantuvo su interés y motivación y propició una mayor interacción virtual con las y los compañeros y la docente, esencial para el aprendizaje de los contenidos.

Respecto del presente, cabe destacar el predominio de la cultura de la información y la comunicación que, aun con sus detractores y defensores, es una realidad de nuestro entorno; por tanto, la educación, en especial la universitaria, debe retomar este desarrollo de la tecnología y la comunicación como un estilo diferente de gestar el proceso de enseñanza en la que se comprenda la relevancia del uso de los entornos virtuales para el logro del aprendizaje significativo (González, 2011).

La inclusión de las TIC en los proceso de enseñanza aprendizaje con estudiantes universitarios abre un mundo de oportunidades en términos de innovación y aplicación para el logro de objetivos concretos y precisos, aunque esta labor no es un fin en sí de las TIC, sino que debe responder a objetivos de aprendizajes claros y concretos; es decir, el uso de las tecnología de la información por sí no es la novedad, sino su uso para un aprendizaje significativo novedoso.

Finalmente, como se menciona en el programa del curso de Anatomía para Enfermería "a través de diversas herramientas metodológicas, se busca que las y los estudiantes adopten un papel activo y protagónico en su proceso de aprendizaje" (p. 3); es decir, el uso de las TIC contribuye a que el alumnado sea el propio constructor de su aprendizaje y no meramente un reproductor del conocimiento dado por otros, como lo expresa magistralmente Gil (2005),

No hay aprendizaje amplio, profundo y duradero sin la participación activa del que aprende. Por lo tanto, no serían admisibles clases en las que los alumnos sean esencialmente receptores pasivos de la información proporcionada por el docente. Esto no quiere decir que no pueda hacerse alguna vez, pero si aceptamos esta característica del aprendizaje significativo en nuestra aula, las clases deberían ser con intensa participación del alumnado (p. 1).

\section{CONCLUSIÓN}

La relación entre los contenidos del curso de anatomía y los diversos escenarios a los que se enfrenta el estudiantado en sus cursos posteriores es fundamental para el logro de un aprendizaje a largo plazo que contribuya a que este sea transferible a otras situaciones de la realidad.

Es clara la resistencia al cambio que se genera tanto en el personal docente como en el alumnado, al incluir las TIC en los cursos, debido a la modificación de los roles que tradicionalmente se han desempeñado: por un lado, el de pasividad por parte del estudiante en cuanto a la producción del proceso de aprendizaje y, por otro, la presencia que se da en el espacio físico del aula, en la que el alumnado ve y escucha al profesorado.

El uso de las TIC en los diversos cursos de la carrera de Licenciatura en Enfermería representa una estrategia novedosa que beneficia el proceso de enseñanza - aprendizaje; sin embargo, es claro que no sustituye al docente, es más, exige no sólo mayor dominio de parte de este en cuanto a las nuevas tecnologías de información, 
sino manejo de la cantidad de datos, capacidad en la selección y calidad de la información de la que dispone el estudiantado para facilitar el aprendizaje significativo.

\section{REFERENCIAS BIBLIOGRÁFICAS}

Ausubel, D., Novak, P. y Hanesian, J. (1978). Educational Psychology. A Cognitive View. 2da. edición. New York: Holt, Rinehart and Winston. Reimpreso, New York: Werbel \& Peck, 1986. Edición en español: Psicología educativa. Un punto de vista cognoscitivo. (1983) México D.F.: Trillas.

Azpilcueta, M. (2002). Herramientas computacionales, multimedia e internet como alternativa educativa en la Unidad Regional Universitaria de Zonas Áridas de la Universidad Autónoma Chapingo. Tercer Congreso Nacional y Segundo Internacional "Retos y Expectativas de la Universidad": Ponencia 81. Universidad Autónoma de México. Recuperado de http://www.congresoretosyexpectativas.udg.mx/Congreso $\% 203 /$ Mesa $\% 205 /$ Mesa5 23.pdf

Ballestero, A. (2002). El aprendizaje significativo en la práctica. Cómo hacer el aprendizaje significativo en el aula. Las Palmas: Ed. Pirámide.

Baños, J. (2007). La plataforma educativa MOODLE: Creación de Aulas Virtuales. España: Creative Commons. Recuperado de http://www.edu.xunta.es/centros/iesasmarinas/aulavirtual/file.php/12/3.Moodle/Material/ManualMoodleIESSatafi Getafe/1_pdfsam_Moodle18_Manual_Prof.pdf.

Bolívar, M. (2009). ¿Cómo fomentar el aprendizaje significativo en el aula? Revista digital para profesionales de la enseñanza N³ 1-6. Recuperado de http://www2.fe.ccoo.es/andalucia/docu/p5sd5097.pdf.

Bouza, O. y Guardado, M. (1999). La informatización: una disciplina necesaria en la educación superior cubana. Revista Pedagogía Universitaria, 4 (2), 57-65. Recuperado de http://cvi.mes.edu.cu/peduniv/index.php/peduniv/article/download/145/145.

Campanario, J. y Otero, J. (2000). Más allá de las ideas previas como dificultades de aprendizaje: las pautas de pensamiento, las concepciones epistemológicas y las estructuras metacognitivas de los alumnos de ciencias. Revista Enseñanza de las Ciencias, 18 (2), 155-169

Cardozo, G. (2008). Tecnologías de la Información y la Comunicación en la Educación. Un punto de vista para discusión. Revista Iberoamericana de Educación, 45(1) 5. Recuperado de http://www.rieoei.org/boletin45 1.htm

Cole, J. y Foster, H. (2008). Using Moodle: teaching with the popular source course management system. 2nd Edition. USA: O”Reilly Media, Inc.

Del Prado, I. (2011). Aprendizaje significativo: David Ausubel. Buenos Aires: Educar el portal educativo de la República de Argentina. 
Dussel, I. y Quevedo, L. (2010). Educación y nuevas tecnologías: los desafíos pedagógicos ante el mundo digital. Buenos Aires: Editorial Santillana.

Falcó, A. (2005). Orientar la asignatura de Anatomía y Fisiología hacia las competencias profesionales de enfermería. Propuesta de un proyecto docente. Revista Educación Médica 8 (4), 208-215.

Galbis, A. (2002). Aprender y Enseñar en Compañía y con Apoyo de TIC: Tecnologías de Información y Comunicación. España: Formación en Economía y Políticas Agrarias y de Desarrollo Rural en América Latina (FODAPAL). Recuperado de http://www.educoas.org/portal/docs/Aprender_enseniar_en_compania.pdf

Gil, N. (2005) “Aprendizaje Significativo”. En Contexto Educativo. Revista Digital de Educación y Nuevas Tecnologías. 36, Año VI. [en línea] www.contexto- educativo.com.ar

González, V. (2011) Aprendizaje significativo y uso de TIC en la Universidad de la Frontera. Rev. Educación y Humanidades 2 (1), Chile.

Gurdián, A. (2001). Las nuevas Tecnologías de la Información y de la Comunicación (NTIC) en la Educación Universitaria. Revista Electrónica Actualidades Investigativas en Educación 1(1), 2-6. Recuperado el 10 de agosto en http://revista.inie.ucr.ac.cr/uploads/tx_magazine/ntic.pdf.

Hernández, R., Fernández, C. y Baptista, P. (2007). Metodología de la Investigación. México D.F.: McGraw Hill.

Inzunza, O. y Bravo, H. (1999). Impacto de dos programas computacionales de Anatomía humana en el rendimiento del conocimiento práctico de los alumnos. Revista chilena de anatomía, 17(2), 205-209. Recuperado de http://www.scielo.cl/scielo.php?script=sci arttext\&pid=S0716-98681999000200012\&lng=es\&tlng=es.

10.4067/S0716-98681999000200012.

Marquéz, P. (2004). Ier Encuentro de profesorado de Centros TIC. Almería: Los cambios que se producen en la escuela con la introducción de las TIC en la sociedad del conocimiento. España: Facultad de Educación, Universidad Autónoma de Barcelona (UAB). Recuperado de http://www.peremarques.net/almeria.htm

Martínez, M. (2000). La investigación acción en el aula. Revista Agenda Académica 7(1), 27- 30.

Meléndez, S. (2009). Mediación Virtual: herramienta para complementar la docencia. Boletín Presencia Universitaria: Universidad de Costa Rica. Recuperado de

http://www.odi.ucr.ac.cr/boletin/index.php?option=com content\&task=view\&id=721\&Itemid=5

Quispe, C. (2005). Eficacia de la educación virtual en el aprendizaje de la anatomía. La Habana: Universidad de la Habana de Cuba. Recuperado de http://www.monografias.com/trabajos40/anatomia-virtual/anatomiavirtual2.shtml 


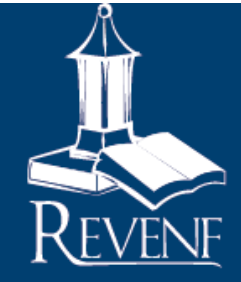

Salinas, J. (2004). Innovación docente y uso de las TIC en la enseñanza universitaria. Revista Universidad y Sociedad del Conocimiento 1(1). 